Mappemonde

Revue trimestrielle sur l'image géographique et les formes du territoire

$131 \mid 2021$

Varia

\title{
Outils pédagogiques innovants dans l'univers Minetest
}

Sivakavi Kumarasamy

Q OpenEdition

Journals

Édition électronique

URL : https://journals.openedition.org/mappemonde/6343

DOI : $10.4000 /$ mappemonde.6343

ISSN : $1769-7298$

Éditeur

UMR ESPACE

\section{Référence électronique}

Sivakavi Kumarasamy, "Outils pédagogiques innovants dans l'univers Minetest », Mappemonde [En ligne], 131 | 2021, mis en ligne le 08 juillet 2021, consulté le 14 juillet 2021. URL : http://

journals.openedition.org/mappemonde/6343; DOI : https://doi.org/10.4000/mappemonde.6343

Ce document a été généré automatiquement le 14 juillet 2021.

\section{(c) (i) (2)(2)}

La revue Mappemonde est mise à disposition selon les termes de la Licence Creative Commons Attribution - Pas d'Utilisation Commerciale - Partage dans les Mêmes Conditions 4.0 International. 


\title{
Outils pédagogiques innovants dans l'univers Minetest
}

\author{
Sivakavi Kumarasamy
}

\section{Contexte}

1 L'Éducation nationale recherche des solutions permettant d'intégrer de plus en plus de contenu numérique dans les salles de classe à des fins pédagogiques. Pour atteindre cet objectif, le ministère de l'Éducation nationale (MEN) a lancé en 2017 l'appel à projets «SINÉ» ("Services innovants numériques Éduthèque »), réservé aux institutions publiques. Le projet "Outils pédagogiques innovants dans l'univers Minetest» (OPIUM), soumis par l'IGN dans ce cadre, a été sélectionné et financé par le MEN avec les fonds du Programme d'investissements d'avenir. Ce projet, lancé en mars 2018 pour 2 ans, englobe notamment le développement d'outils pédagogiques de simulations de risques naturels dans un environnement de jeu de type «bac à sable 3D ", le moteur Minetest (jeu similaire à Minecraft ${ }^{\circledR}$ très connu des enfants et des adolescents).

\section{Service Minetest à la carte}

2 Un premier volet du travail dans ce projet porte sur la mise en place du service Minetest à la carte, réservé au monde de l'éducation, similaire au service gratuit grand public de l'IGN, Minecraft ${ }^{\oplus}$ à la carte. Ce dernier, développé en 2016, permet de générer, avec les données géographiques de l'IGN, en n'importe quel point du territoire national, des cartes au format Minecraft (Lecordix et al., 2017) (Fremont et al., 2016).

3 Ce service a bénéficié initialement des nouveaux développements de l'IGN sur le moteur de calcul des cartes Minecraft et d'interface web afin de répondre à différents besoins pédagogiques identifiés : ajout du format de jeu Minetest, extension du service aux départements et régions d'outre-mer avec les données de l'IGN, puis à la Wallonie avec les données du Service public de Wallonie et au monde entier avec les données d'OpenStreetMap, possibilité de sélectionner des emprises de travail inférieures à $5 \mathrm{~km}$ 
par $5 \mathrm{~km}$ et à des échelles variables ( 1 à 2 cubes par mètre), ajout de l'enneigement potentiel des cartes, etc.

Afin de répondre aux contraintes spécifiques du ministère de l'Éducation nationale, notamment l'intégration de la solution au sein des environnements numériques de travail prévus par le Ministère (accès soit par l'environnement Éduthèque, soit par le gestionnaire d'accès aux ressources numériques), l'interface de génération des cartes Minetest a été placée dans un service spécifique, appelé Minetest à la carte, qui s'est intégré au service Édugéo, déjà proposé par l'IGN pour enseigner avec des données géographiques. Outre la suppression de l'adresse mail dans l'interface de Minetest à la carte, ce service livre, sur la France, en plus de la carte au format Minetest, les images IGN du SCAN $25^{\oplus}$ et aériennes de la $\mathrm{BD} \mathrm{ORTHO}^{\oplus}$ de l'emprise demandée, avec leurs métadonnées de recalage qui vont être exploitées dans la partie suivante du projet (figure 1).

Figure 1. Interface du service « Minetest à la carte » intégré dans Edugéo, permettant de commander une carte Minetest en tout point de la Terre

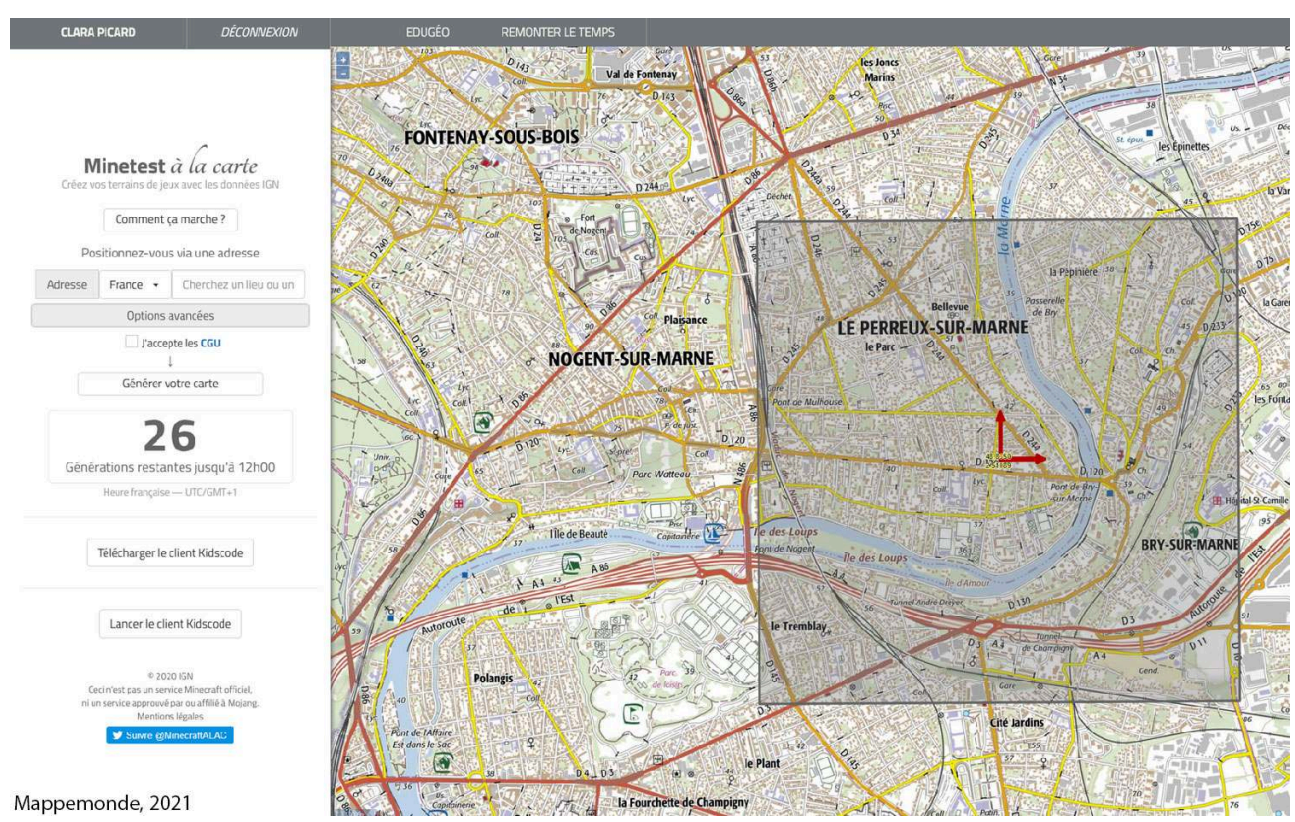

\section{Plateforme pédagogique Kidscode}

5 Le deuxième volet du travail dans ce projet porte sur le développement de la plateforme pédagogique 2D/3D Kidscode qui s'appuie sur le moteur de jeu open source Minetest. Cette partie a été développée par la start-up EvidenceB dans le cadre d'une sous-traitance dirigée par l'IGN, conformément aux contraintes contractuelles liées à l'appel à projets SINÉ (obligation de sous-traitance d'une partie des travaux à une startup).

6 La plateforme Kidscode comporte une interface de visualisation 3D classique pour ce type de jeu bac à sable. Dans le cadre du projet OPIUM, des outils de simulation de risques naturels (inondation, avalanche, coulée de boue, tornade, éruption) ont été ajoutés (figure 2) ainsi que des outils de géovisualisation faisant le lien entre 2D et 3D, avec différents modes de représentation et de localisation dans l'espace géographique. 
7 Cet ensemble d'outils permet aux enseignants d'aborder de manière innovante les programmes liés à l'orientation dans l'espace et aux risques naturels.

Figure 2. Interface de la plateforme 3D Kidscode

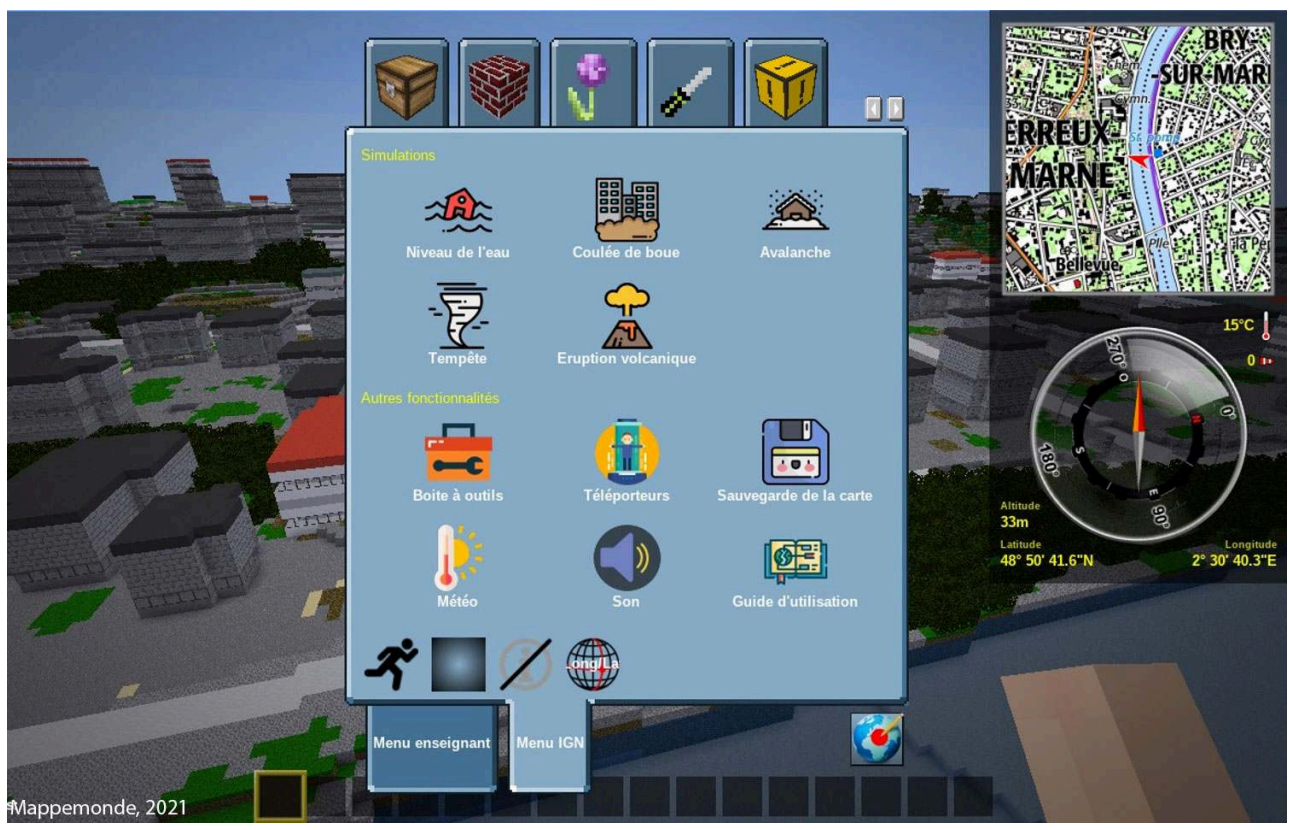

\section{Orientation dans l'espace}

8 Tout d'abord, pour permettre aux élèves de se repérer correctement, la mini-map du jeu, située en haut à droite dans l'interface 3D, affiche, au choix de l'élève, l'aperçu 2D Minetest ou bien les couches IGN raster (SCAN $25^{\oplus}$ ou photographies aériennes), avec une flèche de positionnement de l'élève, afin de mieux se repérer dans l'espace 2D et 3D (figure 3).

9 Une correspondance est réalisée entre coordonnées du jeu et du terrain. L'altitude réelle ainsi qu'une boussole comprenant les quatre points cardinaux sont également disponibles.

10 L'apport des éléments ci-dessus permet de faire un lien par l'espace entre la vision 3D et la cartographie 2D (via la mini-map). Nous avons constaté que les élèves s'orientent facilement dans l'univers cubique 3D Kidscode quand il s'agit de lieux qu'ils connaissent bien (maison, collège, etc.), en comparaison du repérage direct sur une carte 2D. Cela permet ainsi de renforcer l'utilisation de la visualisation 2D en leur offrant deux perspectives simultanées.

11 Avec la boussole et l'évolution en temps réel des coordonnées, ils peuvent facilement situer un point précis à partir de ses coordonnées. Enfin, la possibilité d'afficher les coordonnées géographiques (longitudes, latitudes) ou cartographiques (Lambert 93) leur permet d'étudier les différentes possibilités de représentation de la Terre via les projections. 
Figure 3. Lien entre visualisation 3D dans le jeu et 2D dans la carte SCAN 25® avec la correspondance entre coordonnées en jeu et coordonnées géographiques (longitude/latitude ou Lambert 93)

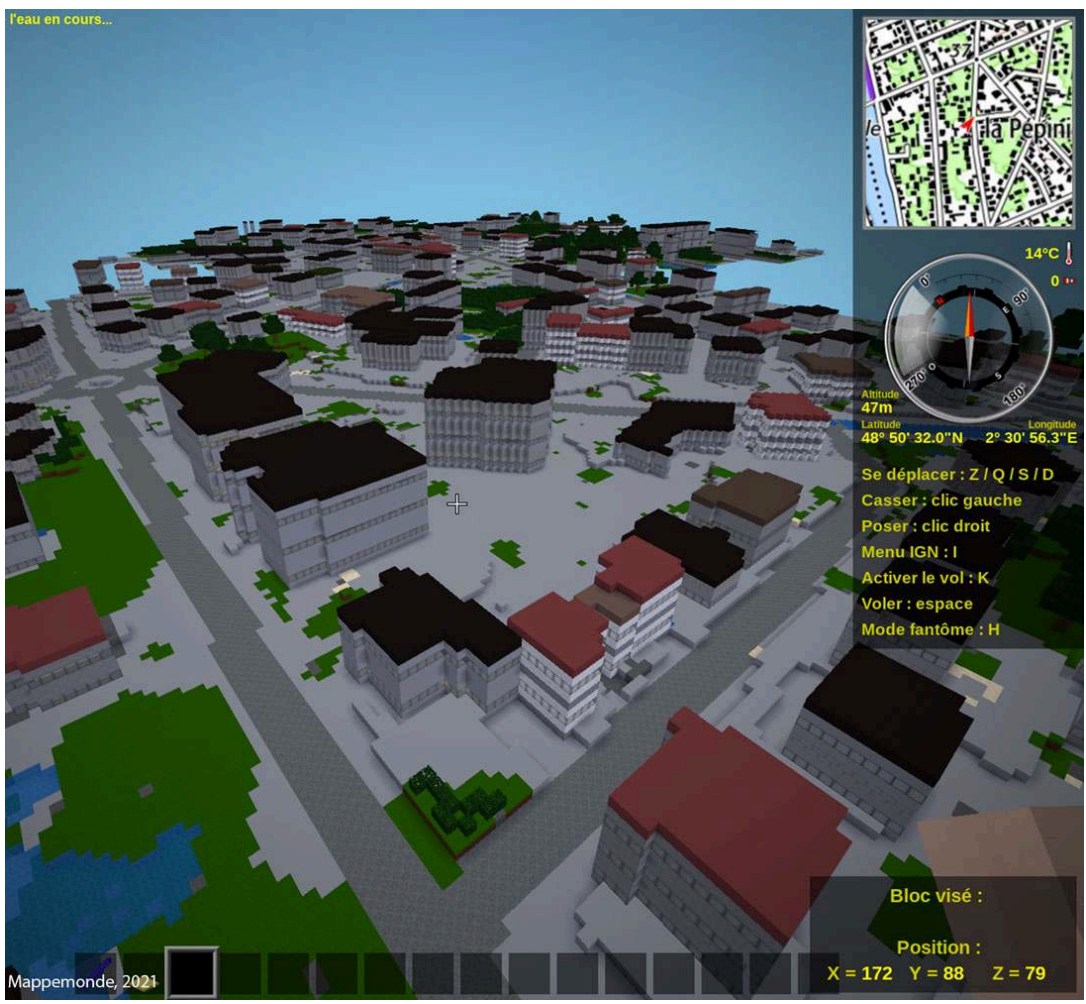

\section{Simulation de risques naturels en 3D}

12 L'autre outil de géovisualisation nouveau mis en place lors du projet OPIUM concerne la simulation de risques naturels sur la plateforme Kidscode dans l'environnement du jeu Minetest.

Après avoir obtenu la carte Minetest en n'importe quel lieu grâce au service Minetest à la carte, il est possible d'activer sur la plateforme Kidscode six types de simulations de risques naturels : inondation, avalanche, coulée de boue, éruption volcanique, tornade. Ces simulations permettent de visualiser les conséquences de ces risques naturels dans l'environnement géographique cartographié, comme le montre la figure 4 pour le risque d'inondation avec la montée des eaux de la Marne sur la commune du Perreux.

La plateforme permet d'annuler les simulations, puis de les relancer avec d'autres paramètres. Elle permet aussi d'intervenir sur les cartes pour proposer des solutions de protection contre ces risques, par exemple en construisant dans les cartes 3D des digues pour les inondations ou des paravalanches pour les avalanches. 
Figure 4. Visualisation de la simulation d'inondation de la Marne au Perreux-sur-Marne dans Minetest en 3D

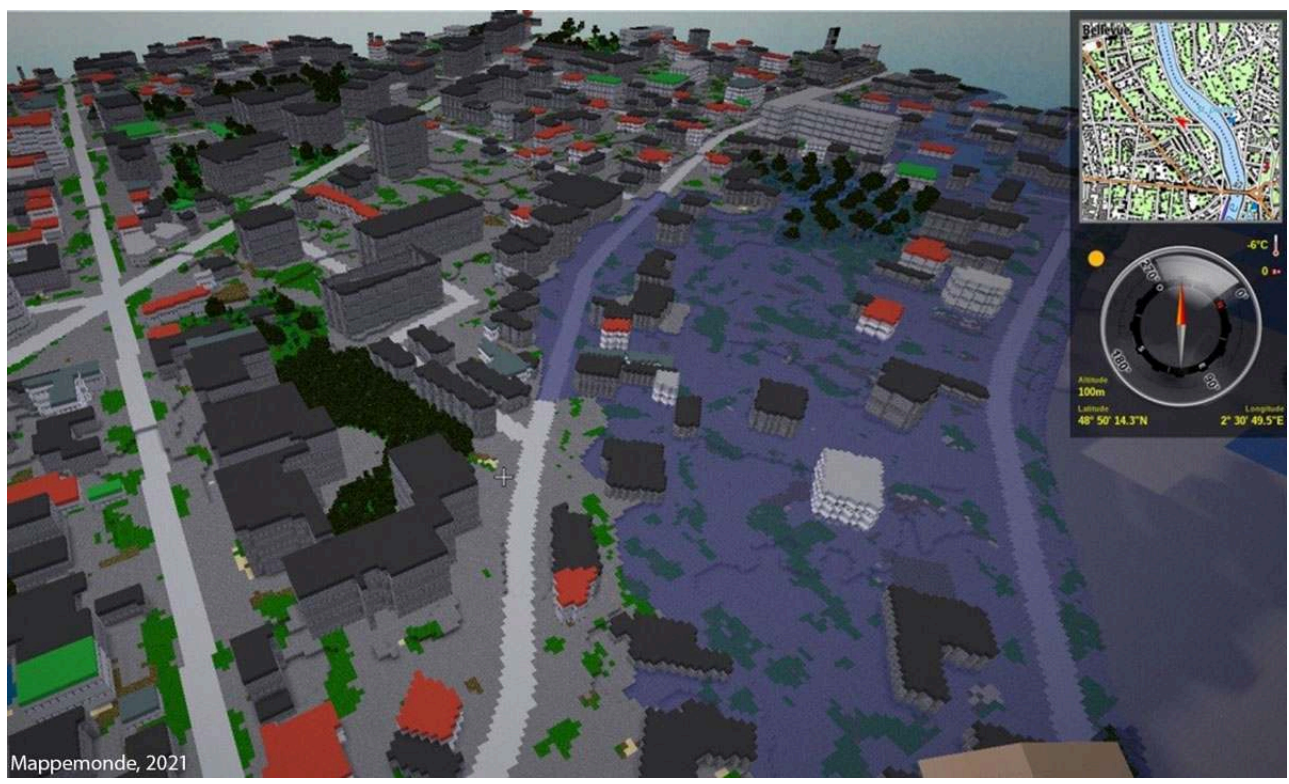

\section{Cartographe 2D}

En plus de la visualisation en 3D dans la plateforme Kidscode, l'IGN a piloté le développement d'un outil de géovisualisation 2D, le Cartographe, particulièrement destiné au cas des simulations d'inondation. Cet outil de visualisation 2D est un fork ${ }^{1}$ Kidscode qui a été développé sur la base de "Minetest Mapserver». Il s'agit d'un serveur développé en Go qui se branche directement sur les cartes Minetest (écrites dans une base de données fichier en SQLite).

Cet outil assure la mise à jour en temps réel de l'aperçu 2D via ce qu'on appelle un "rendering incrémental». Chaque chunk (ensemble de blocs) de la carte Minetest constitue une ligne en base de données, avec, entre autres, une colonne "mtime» servant d'horodatage et permettant de déterminer les changements récents à calculer.

Les ajouts importants en termes de géovisualisation ont été l'implémentation complète d'outils de cartographie (légende, coloration, zones...) ainsi que la superposition avec transparence des couches IGN SCAN $25^{\oplus}$ et BD $\mathrm{ORTHO}^{\circledR}$ (figure 5) ce qui permet aux élèves de travailler sur un rendu 2D des simulations et de fournir des comparaisons, analyses et synthèses. 
Figure 5a. Outil Cartographe 2D à différents niveaux de zoom et avec différentes options de transparence et de fonds de carte (carte Minetest)

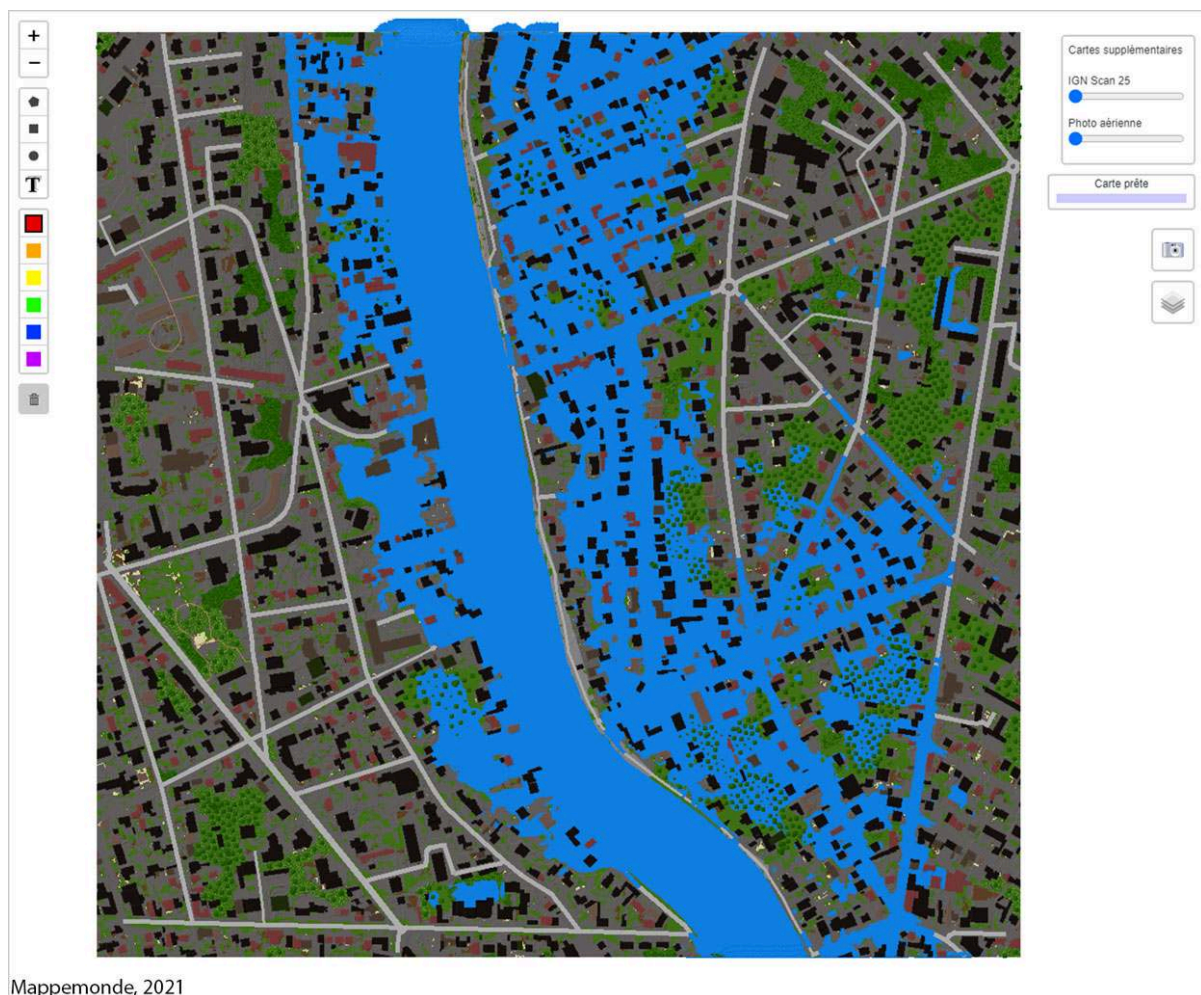

Figure $5 \mathrm{~b}$. Outil Cartographe 2D à différents niveaux de zoom et avec différentes options de transparence et de fonds de carte (SCAN 25®)

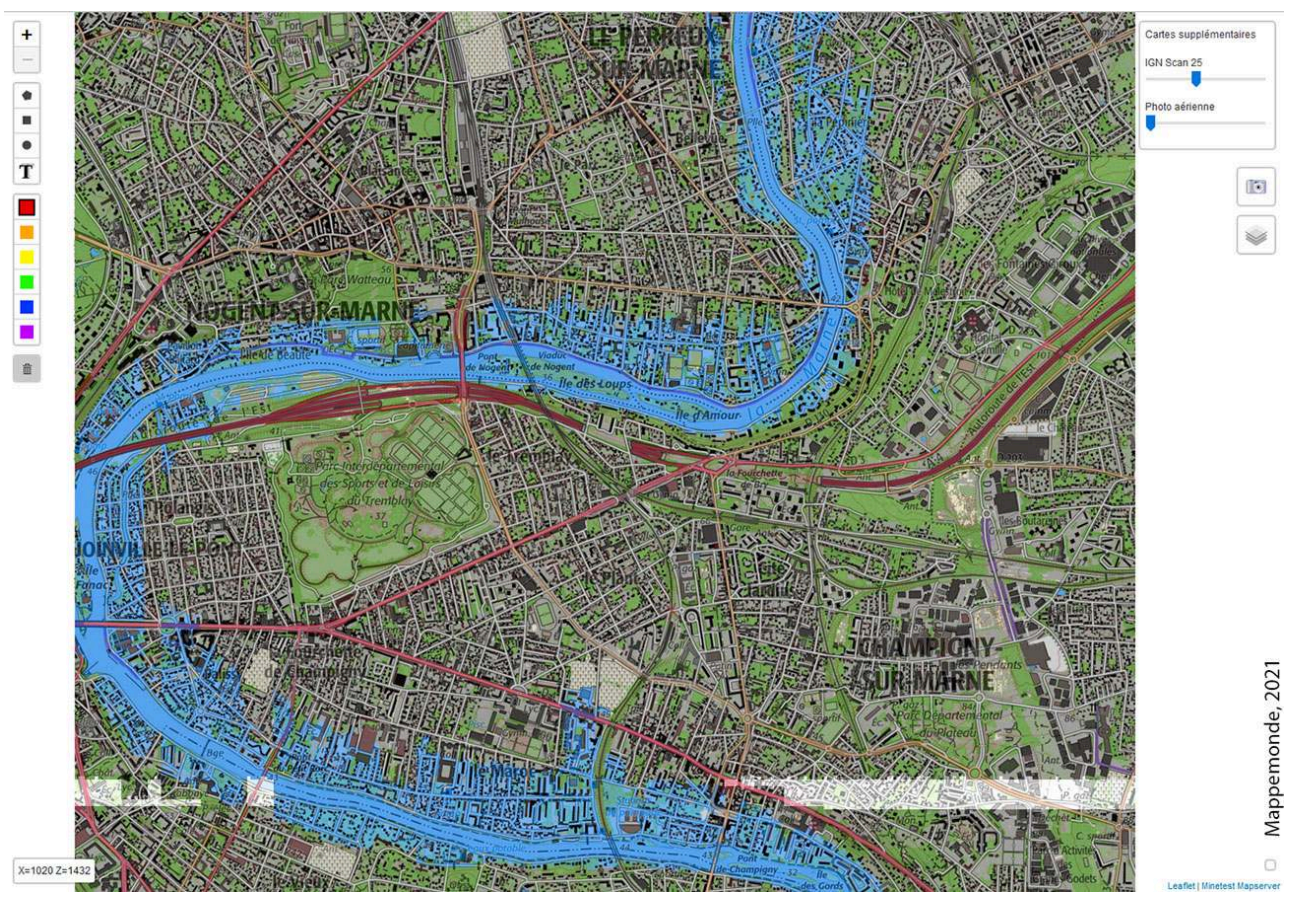

18 Au-delà du travail dans son environnement proche (son école, sa maison, etc.), ces outils de simulation et de cartographie peuvent permettre aussi d'étudier des évènements d'actualité, comme la tempête Xynthia, ou plus anciens comme la crue de 
1910 en région parisienne. Ainsi, pour la carte de la figure 5, il est intéressant de comparer avec la crue de 1910 dont les cartographies des zones d'inondation sont disponibles sur le Géoportail.

Figure 6. Visualisation de la crue de la Marne en 1910 disponible sur le site du Géoportail comparable avec les simulations obtenues dans Kidscode

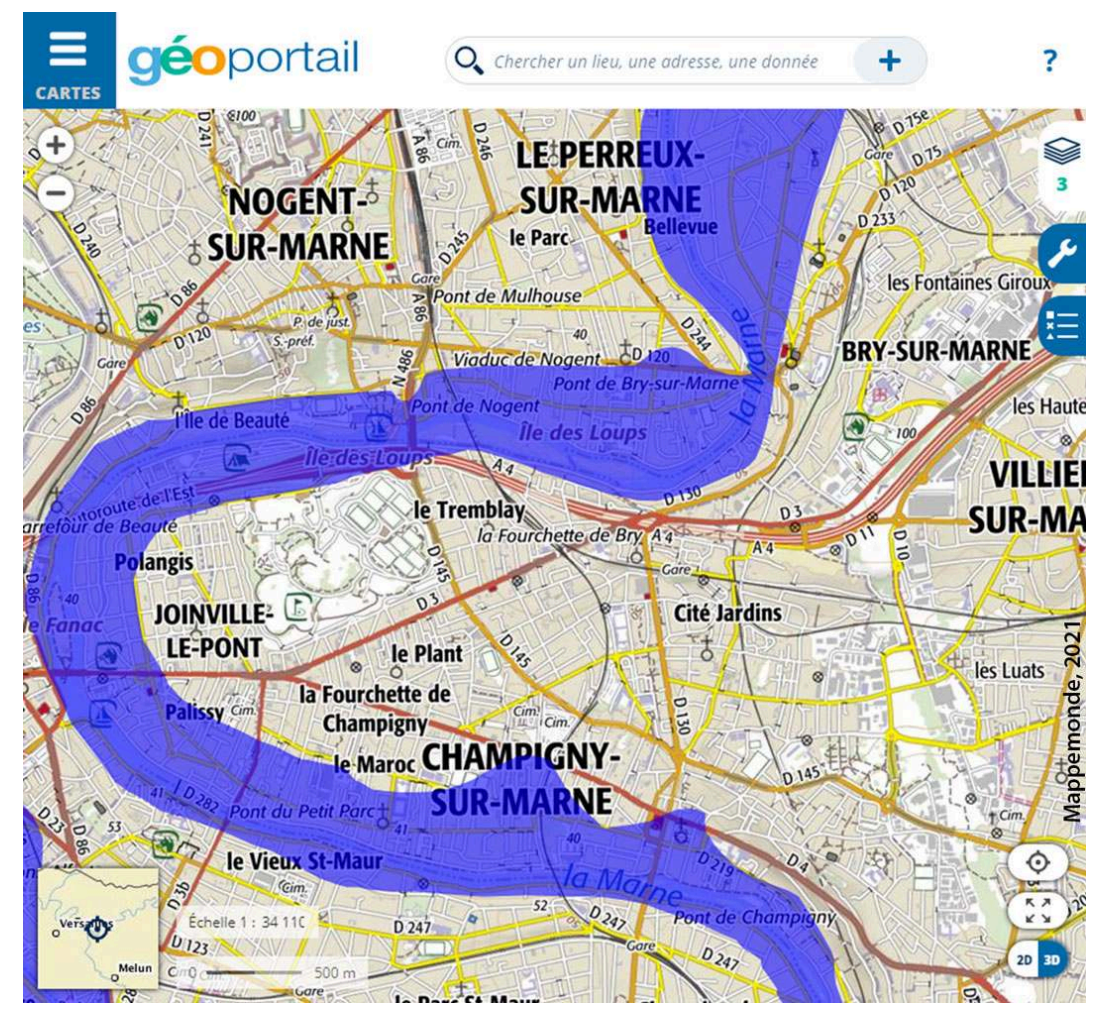

cf. figure 5 .

\section{Conclusion}

Afin de répondre au besoin du ministère de l'Éducation nationale d'intégration de plus de contenu numérique dans les salles de classe à des fins pédagogiques, le projet OPIUM a apporté des outils nouveaux de simulations de risques naturels et de géovisualisation s'intégrant dans la plateforme Kidscode de type "jeu bac à sable», sur la base du moteur Minetest.

Cet objectif prend encore plus son sens avec la pandémie actuelle de COVID-19 et son impact via l'enseignement à distance obligatoire instauré. S'il est trop tôt pour observer si la solution proposée sera adoptée par les enseignants, elle offre cependant une opportunité pour donner aux élèves l'accès à leur premier Système d'Information Géographique (SIG), avec des outils de manipulation et de géovisualisation des données géographiques, et peut-être pour susciter des vocations de futurs géomaticiens.

De plus, les outils proposés pourraient être exploités plus largement pour la sensibilisation du grand public aux risques d'inondation de leur environnement. La plateforme de simulation Kidscode a déjà montré son potentiel d'exploitation pour l'examen de la crue lente de plaine avec, par exemple, le cas de Paris en 1910 abordé précédemment. 
Il serait intéressant d'expérimenter sur d'autres types d'inondations cartographiées (submersion marine, remontée de nappe, crue torrentielle liée à des averses comme celle de la Vésubie...) afin de couvrir un plus grand nombre de cas et sensibiliser plus fortement la population à ce risque qui concerne une commune sur deux en France. Pour cela, des adaptations et améliorations de simulation seront sans doute nécessaires et pourront faire l'objet de travaux futurs.

\section{BIBLIOGRAPHIE}

FREMONT D., JILANI M., SÉGUIN E., LECORDIX F., KRIAT S. (2017). « Minecraft ${ }^{\circledR}$ à la carte. Le nouveau service de l'IGN qui allie jeu vidéo et cartographie 3D ». Mappemonde, $\mathrm{n}^{\circ} 120$. En ligne : http:// mappemonde.mgm.fr/120geov2/

LECORDIX F., FREMONT D., JILANI M., SÉGUIN E., KRIAT S. (2018). “Minecraft ${ }^{\oplus}$ on Demand. A new IGN service which combines game and 3D cartography”. Proceedings of the ICA, vol. 1, p. 1-5. En ligne : https:// www.proc-int-cartogr-assoc.net/1/65/2018/

\section{NOTES}

1. Un fork (de l'anglais signifiant «fourche », « bifurcation ») désigne un nouveau logiciel créé à partir du code source d'un logiciel existant.

\section{AUTEUR}

SIVAKAVI KUMARASAMY

ENSIIE/IGN, Saint-Mandé 